\title{
Daily peak load forecast using artificial neural network
}

\author{
Ramesh Kumar V' ${ }^{1}$, Pradipkumar Dixit ${ }^{2}$ \\ ${ }^{1}$ Department of Electrical and Electronics Engineering, JAIN (Deemed-to-be-University), India \\ ${ }^{2}$ Department of Electrical and Electronics Engineering, M. S. Ramaiah Institute of Technology, India
}

\begin{tabular}{l}
\hline \hline Article Info \\
\hline Article history: \\
Received Sep 5, 2018 \\
Revised Dec 27, 2018 \\
Accepted Jan 10, 2019 \\
\hline
\end{tabular}

Keywords:

Artificial Neural Network

Daily peak load

Forecasting

Linear fit

Polynomial fit

\begin{abstract}
The paper presents an Artificial Neural Network (ANN) model for short-term load forecasting of daily peak load. A multi-layered feed forward neural network with Levenberg-Marquardt learning algorithm is used because of its good generalizing property and robustness in prediction. The input to the network is in terms of historical daily peak load data and corresponding daily peak temperature data. The network is trained to predict the load requirement ahead. The effectiveness of the proposed ANN approach to the short-term load forecasting problems is demonstrated by practical data from the Bangalore Electricity Supply Company Limited (BESCOM). The comparison between the proposed and the conventional methods is made in terms of percentage error and it is found that the proposed ANN model gives more accurate predictions with optimal number of neurons in the hidden layer.
\end{abstract}

Copyright $(2019$ Institute of Advanced Engineering and Science. All rights reserved.

\section{Corresponding Author:}

Ramesh Kumar V,

Research Scholar, JAIN (Deemed-to-be-University),

Department of Electrical and Electronics Engineering,

JGI Global Campus, Bengaluru-562 112, Karnataka State, India.

Email: ramesh_uvce@rediffmail.com

\section{INTRODUCTION}

Electrical energy plays a vital role in day to day life. It is required for domestic purpose, industrial purpose and in many areas. Electrical energy is in great demand by consumers. At present, in India according to Central Electricity Authority, New Delhi survey [1] the total installed capacity as on 31-03-2017 is $326.848 \mathrm{GW}$ and has energy shortage of $0.7 \%$ and peak shortage of $1.6 \%$ during the financial year 2016-17 and similarly in Karnataka the total installed capacity is $21.316 \mathrm{GW}$ and has energy shortage of $0.5 \%$ and peak shortage of $0.2 \%$. The shortage of power is because of lack of generation due to shortage of resources, unscheduled maintenances, outages and faults. This deficit can be reduced to some extent by demand side management or by transmitting power at high voltages which reduces transmission losses, but the basic means of meeting the demand is by increasing the generation itself. For this purpose, prediction of future load requirement is essential. Determining an estimate of load requirements for future is known as load forecasting. Depending on the duration, load forecasting is generally classified into short-term, medium-term and long-term [2].

The development of an accurate, fast and robust electrical load forecasting methodology is of importance to both the electric utility and its customers. A forecast that is too low or too high can result in revenue loss [3]. A wide variety of forecasting models have been proposed in the literature, most of which can be generally classified into two broad categories: 1) Statistical or Classical approaches such as multiple regression, exponential smoothing, minimum mean square estimation, Box and Jenkins methods, Kalman filter and state estimation. 2) Artificial Intelligence (AI) based methods such as Expert system, Neural Networks, Fuzzy Logic, Particle Swarm Optimizer (PSO), Support Vector Machine (SVM) and Genetic Algorithm (GA). Other category such as hybrid techniques is a combination of more than one technique i.e., either the combination of classical and AI techniques or the combination of different AI techniques [4]. 
Tomonobu Senjyu et al. [5], employed ANN with back propagation algorithm to forecast the load by adding a correction to the selected similar day data. Load deviation and temperature deviation data is used as the correction for forecasting load. If the temperature curves change rapidly on the forecast day, load power changes greatly and forecast error would increase. Vitor Hugo Ferreira et al. [6], proposed ANN model with Bayesian training and SVM learning algorithm to control the ANN complexity. The three input datasets used were hourly load and temperature, daily peak load and temperature, and half-hourly load temperature and price. Z. A. Bashir et al. [7], implemented adaptive artificial neural networks to predicted hourly load demand and trained the ANNs with PSO algorithm with load, temperature, wind speed, humidity as input data. Ying Chen et al. [8], developed wavelet based neural network method to forecast next day demand, with similar day load and weather information such as wind-chill temperature, humidity, wind speed, cloud cover, and precipitation as inputs. Madasu Hanmandlu et al. [9], proposed hybrid neural networks to forecast hourly load demand, with load data and weather data comprising temperature, wind speed and relative humidity. Ni Ding et al. [10], proposed Generalized Neural Network model, with load data, temperature data and cost function (minimization) as input parameters. Yizheng Xu et al. [11], applied ANN and Monte Carlo Simulations technique to predict load a day ahead using load data, temperature, humidity and wind speed as input variables. Filipe Rodrigues et al. [12], proposed Feed-forward ANN with the Levenberg-Marquardt learning algorithm. Input variables are area location, number of consumers and consumption of electrical appliance with hourly load consumption were considered to forecast the residential demand. Anamika et al. [13], proposed multilayer feed forward network with ten hidden layers and Levenberg Marquardt back propagation learning algorithm for training the network. The input to the network is half hourly load data.

The above work [5-11], employs either hybrid techniques that is the combination of different AI techniques or combination of classical and AI techniques or utilizes more than two input parameters to forecast the load demand and [12-13], though employs ANN techniques with Levenberg-Marquardt learning algorithm but does not provides any information regarding the number of neurons in the hidden layer(s) that represents the actual ANN model. Hence, the present work proposes an ANN model that uses load and/or temperature data for training the network to predict daily peak load demand with optimal number of hidden layer neurons and is validated on BESCOM power systems.

\section{CONVENTIONAL TECHNIQUES}

The simplest conventional method employed for load forecasting is curve fitting techniques. The procedure of determining the empirical equation of the curve of best fit is known as curve fitting techniques. Some of the curve fit techniques used in electrical load forecasting are

- $\quad$ Linear: $y=A x+B$

- Exponential: $y=A e^{B x}$

- Logarithmic: $y=A \ln (x)+B$

- Polynomial: $y=A x^{2}+B x+C$

- $\quad$ Power: $y=A x^{B}$

Where $\mathrm{A}, \mathrm{B}$ and $\mathrm{C}$ are the constants determined by principle of least squares. Let $x$ be the independent variable that represents days of a month and $\mathrm{y}$ be the dependent variable on $x$ representing the forecasted demand such that $y=f(x)$. Now consider the $\mathrm{n}^{\text {th }}$ polynomial function

$$
f(x)=a_{0}+\sum_{i=1}^{n} a_{i} x^{i}
$$

In (1), the constant $a_{0}$ is the interceptor of y-axis which represents the base load. In present work, for $i=1,2$ the other constants $a_{1}$ and $a_{2}$ are the exogenous factors. With the known parameters $y$ (historical load) and $x$ (day) the best fit can be done to evaluate these constants with least error.

\section{ARTIFICIAL NEURAL NETWORK TECHNIQUES}

A fully connected multilayer feed-forward neural network model shown in Figure 1 is employed for daily peak load forecasting of the month. The input layer consists of 24 nodes corresponding to two input parameters viz., 12 input nodes, each node representing a month and has a maximum of 31 daily peak load data represents the corresponding days of a particular month and similarly another 12 input nodes for daily peak temperature data. The output layer consists of 12 nodes each node corresponds to a month and has a maximum of 31 daily peak forecasted load representing days of the month. The single hidden layer is used with variable number of nodes to verify the dependency upon the correct percentage of forecasting and repeatability in convergence. The output function of each unit in the hidden and output layers is non-linear 
(sigmoid). Inputs to the proposed model are $a_{1}, a_{2} \ldots a_{12}$, daily peak load growth data and $a_{13}, a_{14} \ldots a_{24}$, daily peak temperature data of the corresponding month. The outputs of input layer for $i=1,2 \ldots 24$ is given by (2).

$$
s_{i}=a_{i}
$$

The outputs of the hidden layer $\left(s_{j}^{h}\right)$ and output layer $\left(b_{k}\right)$ are represented by (3) and (4) respectively for $j=1,2 \ldots \mathrm{m}$ and $k=1,2 \ldots 12$

$$
\begin{aligned}
& s_{j}^{h}=f\left\{\sum_{i=1}^{24} W_{j i}^{h} s_{i}-\theta_{j}^{h}\right\} \\
& b_{k}=f\left\{\sum_{j=1}^{m} W_{k j}^{o} s_{j}^{h}-\theta_{k}^{o}\right\}
\end{aligned}
$$

Where, $W_{j i}^{h}$ and $W_{k j}^{o}$ represents weights of the links for input-hidden layers and hidden-output layers respectively and $\theta_{j}^{h}$ and $\theta_{k}^{o}$ are the bias terms of hidden and output layers respectively. The outputs of the network correspond to $b_{1}, b_{2} \ldots b_{12}$ are the daily peak load forecast of the corresponding month.

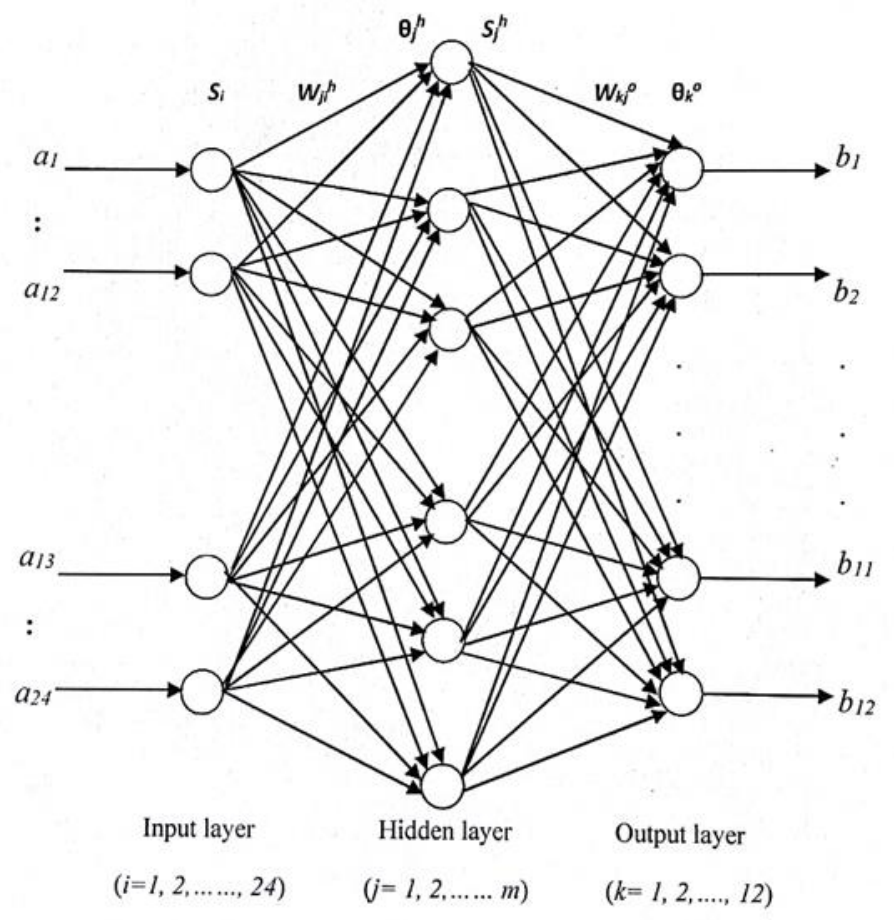

Figure 1. Multilayer feed-forward neural network model for daily peak load forecast

\section{INPUT DATA SELECTION}

In the present work, the hourly peak load data for more than five years i.e., from Jan 2012 to March 2017 is collected from Bangalore Electricity Supply Company and the hourly peak temperature data from Jan 2016 to March 2017 is collected from Meteorological Centre, Bangalore. Using the daily peak load data from 2012 to 2016, the average load data for 2017 is calculated to obtain the empirical curve fit equation in conventional method. For ANN technique, the load growth data for 2017 is estimated, with the load growth data of 2017 and the temperature data, the daily peak load as per the requirements can be forecasted ahead.

Normalization of data, the input data for the neural network will have very wide ranges if the actual load data is directly used. This may cause convergence problem [14] during the learning process. To avoid this, the input data were normalized such that they were within the range of 0 to 1 . For this purpose, the load and temperature data are normalized using (5). 


$$
L_{n o r}=\frac{L_{(i, j)}}{L_{j_{\max }}}
$$

Where, $L_{\text {nor }}$ is the normalized load/temperature data which is used as input to the net, $L_{(i, j)}$ is the actual load growth/temperature data, $i=1$ to $\mathrm{n}, \mathrm{n}$ (number of days) $=1,2 \ldots 31$ and $j=1$ to $\mathrm{m}, \mathrm{m}$ (number of months) $=1,2, \ldots . .12$ and $L_{j_{\max }}$ is the maximum load/temperature of a particular month. Thus, the input matrix will be in the order of $31 \times 12$ and using these data the daily peak load for any particular month or complete year can be forecasted.

\section{TRAINING THE NETWORK}

Training of the network is completely based on the actual load data and temperature data collected from the respective authority on hourly basis. From the collected data, the data for more than five years have been analyzed for the percentage growth from year to year, month to month and hour to hour. After understanding the percentage growth, the training file for the neural network has been prepared and used to predict the future load demand. The size of the training file with temperature parameter included, for entire year will be in the order of 31×24 and testing file size can be same as that of training file or subset of training file as per the demand to be forecasted.

The network is trained using Levenberg-Marquardt training algorithm for 2000 epochs and threshold on error, set to a very low value of $10^{-5}$. The learning rate parameter and momentum factors are 0.1 and 0.9 respectively [15]. Training is carried out until the total sum of mean squares error reaches either the desired error limit of $10^{-5}$ or till the completion of 2000 epochs. The network was initially trained with varying number of nodes in the hidden layer. The smallest sum of squares error $\left(10^{-5}\right)$ was obtained for the network structure with $24 N_{i}, 21 N_{h}$ and $12 N_{o}$ input layer, hidden layer and output layer respectively. Figure 2 shows the trend in the training error for each epoch for 21 nodes in the hidden layer for March 2017.

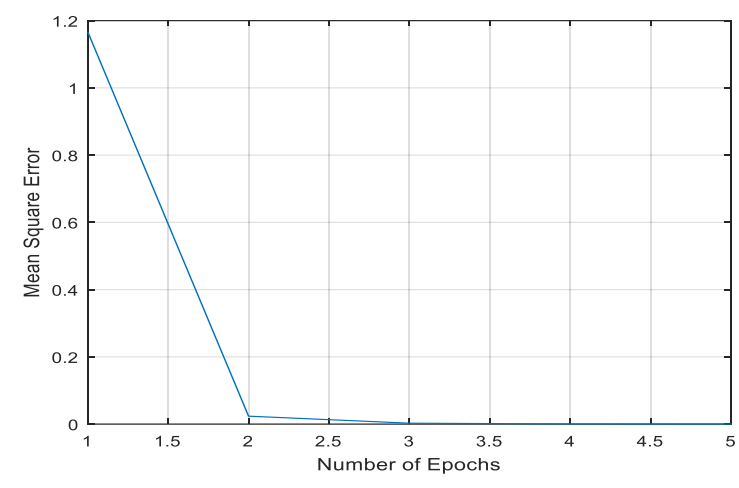

Figure 2. Training error curve for March 2017

\section{RESULTS AND DISCUSSIONS}

\subsection{Demand forecasting using conventional methods}

In this method, the trend in the historical load data points is plotted with the use of linear and polynomial of second order function to obtain the equation of empirical curve fit. The coefficients values of the fitted curve are given by least square approach. The error will minimum for the best fit, when the goodness of fit i.e. $\mathrm{R}^{2}$ tends towards one. The coefficient $a_{0}$ represents the base load and other $a_{1}, a_{2}$ represents the exogenous factors. The linear and polynomial fit for March 2017 is shown in Figure 3 and the corresponding empirical curve fit equation is given by (6a) and (6b), respectively.

$$
\begin{aligned}
& Y=3.7762 x+4011.8 M W \\
& Y=-0.1816 x^{2}+9.5869 x+3979.8 M W
\end{aligned}
$$

Where, $\mathrm{Y}$ is forecasted load in MW and $\mathrm{x}$ is the day of the month.

The demand forecast calculated by using above equations and percentage error is calculated using (7) is tabulated in Table 1. The result shows that forecasted values by these methods has large error due 
to the non-linearity of load. The linear fit has the maximum error of $13 \%$ and minimum of $2.82 \%$, while polynomial fit has maximum of $13.06 \%$ and minimum of $2.55 \%$ error of actual demand. The plot of actual demand against the forecasted load of linear and polynomial fit is shown in Figure $4 \mathrm{a}$ and $4 \mathrm{~b}$ respectively. This method requires one equation for a month and 12 equations for a year and hence the process becomes complex and tedious. Further the number of equations required can be reduced to one equation for a year by taking average of corresponding daily peak load over a complete year. With the reduction in number of equations, the percentage error increases drastically.

$$
\% \text { Error }=\left|\frac{\text { Load }_{\text {Actual }}-\text { Load }_{\text {Forecasted }}}{\text { Load }_{\text {Actual }}}\right| \times 100
$$

Table 1. Daily load forecast results for March 2017

\begin{tabular}{|c|c|c|c|c|c|c|c|c|c|}
\hline \multirow[b]{2}{*}{ Day } & \multirow[b]{2}{*}{$\begin{array}{l}\text { Actual } \\
\text { Load } \\
(\mathrm{MW})\end{array}$} & \multirow[b]{2}{*}{$\begin{array}{c}\text { Forecasted } \\
\text { Load (MW) } \\
\text { with linear fit }\end{array}$} & \multicolumn{3}{|c|}{ Forecasted } & \multicolumn{4}{|c|}{ ANN Forecasted Load (MW) } \\
\hline & & & $\begin{array}{c}\% \\
\text { Error }\end{array}$ & $\begin{array}{c}\text { Load (MW) } \\
\text { with } \\
\text { Polynomial fit }\end{array}$ & $\begin{array}{c}\% \\
\text { Error }\end{array}$ & $\begin{array}{c}\text { Without } \\
\text { Temperature }\end{array}$ & $\begin{array}{c}\% \\
\text { Error }\end{array}$ & $\begin{array}{c}\text { With } \\
\text { Temperature }\end{array}$ & $\begin{array}{c}\% \\
\text { Error }\end{array}$ \\
\hline 1 & 4438 & 4016 & 9.52 & 3989 & 10.11 & 4471 & -0.74 & 4456 & -0.40 \\
\hline 2 & 4490 & 4019 & 10.48 & 3998 & 10.95 & 4560 & -1.57 & 4536 & -1.02 \\
\hline 3 & 4527 & 4023 & 11.13 & 4007 & 11.49 & 4545 & -0.40 & 4537 & -0.22 \\
\hline 4 & 4499 & 4027 & 10.49 & 4015 & 10.75 & 4619 & -2.68 & 4609 & -2.44 \\
\hline 5 & 4246 & 4031 & 5.07 & 4023 & 5.25 & 4420 & -4.10 & 4413 & -3.94 \\
\hline 6 & 4419 & 4034 & 8.70 & 4031 & 8.79 & 4298 & 2.73 & 4297 & 2.76 \\
\hline 7 & 4417 & 4038 & 8.58 & 4038 & 8.58 & 4520 & -2.34 & 4511 & -2.12 \\
\hline 8 & 4455 & 4042 & 9.27 & 4045 & 9.21 & 4365 & 2.01 & 4359 & 2.15 \\
\hline 9 & 4420 & 4046 & 8.47 & 4051 & 8.34 & 4529 & -2.47 & 4513 & -2.11 \\
\hline 10 & 4431 & 4050 & 8.61 & 4058 & 8.43 & 4582 & -3.40 & 4564 & -3.01 \\
\hline 11 & 4390 & 4053 & 7.67 & 4063 & 7.44 & 4575 & -4.21 & 4564 & -3.96 \\
\hline 12 & 4175 & 4057 & 2.82 & 4069 & 2.55 & 4368 & -4.61 & 4359 & -4.40 \\
\hline 13 & 4296 & 4061 & 5.47 & 4074 & 5.17 & 4467 & -3.97 & 4448 & -3.53 \\
\hline 14 & 4249 & 4065 & 4.34 & 4078 & 4.01 & 4452 & -4.78 & 4433 & -4.32 \\
\hline 15 & 4546 & 4068 & 10.50 & 4083 & 10.19 & 4328 & 4.81 & 4426 & 2.65 \\
\hline 16 & 4523 & 4072 & 9.97 & 4087 & 9.65 & 4336 & 4.14 & 4433 & 2.00 \\
\hline 17 & 4550 & 4076 & 10.41 & 4090 & 10.09 & 4443 & 2.33 & 4438 & 2.46 \\
\hline 18 & 4605 & 4080 & 11.41 & 4094 & 11.11 & 4508 & 2.10 & 4507 & 2.12 \\
\hline 19 & 4324 & 4084 & 5.56 & 4096 & 5.26 & 4475 & -3.49 & 4465 & -3.26 \\
\hline 20 & 4667 & 4087 & 12.42 & 4099 & 12.17 & 4623 & 0.95 & 4621 & 0.98 \\
\hline 21 & 4541 & 4091 & 9.91 & 4101 & 9.69 & 4713 & -3.78 & 4684 & -3.15 \\
\hline 22 & 4707 & 4095 & 13.00 & 4103 & 12.84 & 4722 & -0.33 & 4712 & -0.12 \\
\hline 23 & 4554 & 4099 & 10.00 & 4104 & 9.88 & 4728 & -3.83 & 4721 & -3.67 \\
\hline 24 & 4635 & 4102 & 11.49 & 4105 & 11.43 & 4667 & -0.68 & 4657 & -0.48 \\
\hline 25 & 4626 & 4106 & 11.24 & 4106 & 11.24 & 4516 & 2.37 & 4516 & 2.37 \\
\hline 26 & 4435 & 4110 & 7.33 & 4106 & 7.41 & 4406 & 0.65 & 4404 & 0.69 \\
\hline 27 & 4723 & 4114 & 12.90 & 4106 & 13.06 & 4573 & 3.18 & 4670 & 1.12 \\
\hline 28 & 4652 & 4118 & 11.49 & 4106 & 11.74 & 4605 & 1.02 & 4606 & 1.00 \\
\hline 29 & 4434 & 4121 & 7.05 & 4105 & 7.42 & 4620 & -4.20 & 4593 & -3.58 \\
\hline 30 & 4434 & 4125 & 6.97 & 4104 & 7.44 & 4610 & -3.98 & 4594 & -3.62 \\
\hline 31 & 4605 & 4129 & 10.34 & 4102 & 10.91 & 4663 & -1.25 & 4641 & -0.79 \\
\hline
\end{tabular}

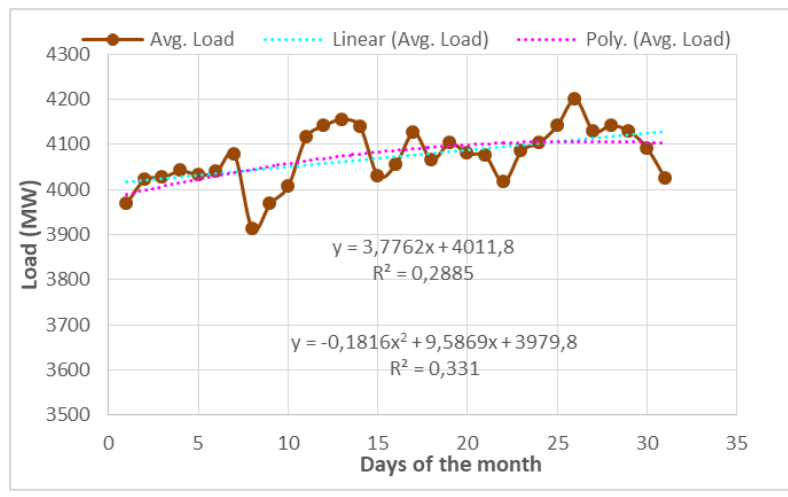

Figure 3. Linear fit and polynomial fit for March 2017 


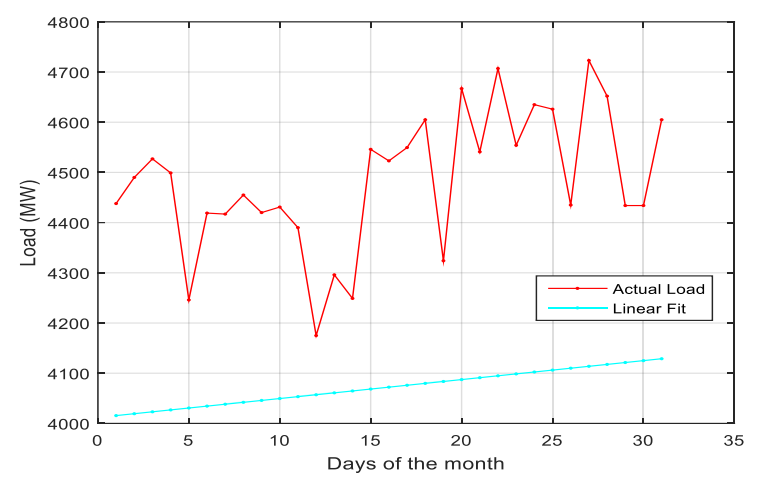

(a)

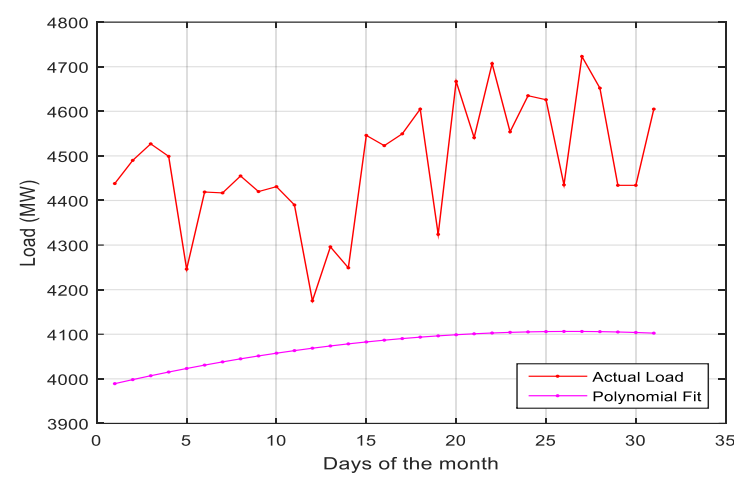

(b)

Figure 4. a. Comparison of actual and forecasted load curve of linear fit for March 2017

b. Comparison of actual \& forecasted load curve of polynomial fit for March 2017

\subsection{Demand forecasting using developed ANN model}

MATLAB code is written to train and test the proposed ANN model to forecast the demand. The performance of the ANN model has been tested for March 2017. The percentage of correct prediction obtained from the ANN model with varying number of nodes in the hidden layer has been performed. It is observed that the network structure with 21 nodes in the hidden layer gave the most accurate forecast, trained with temperature as shown in Table 2. The forecasted daily peak load for March 2017 is compared with the actual load data and shown in Figure 5. The percentage error calculated using (7) is tabulated in Table 1. The proposed ANN model, when trained with only load data as its input parameter predicted the demand with maximum percentage error of 4.81 and minimum of -0.33 . The same ANN model on training with both load and temperature data as its input parameters gave further accurate prediction with maximum of $-4.40 \%$ error and minimum of $-0.12 \%$ error as shown in Table 1 . The results show that one of the influential factors such as temperature has an impact on the demand forecast. Figure 6 shows the comparison of forecasted demand by conventional methods and proposed ANN model. The ANN model gives more accurate prediction as compared to the empirical curve fitting technique and also it can handle large number of nonlinear load data with ease. Using the same training file the proposed ANN model can forecast the daily peak demand for complete year.

Table 2. Maximum percentage error for different Neural Network structure

\begin{tabular}{ccccc}
\hline SL No & $\begin{array}{c}\text { No of Input } \\
\text { Layer Neurons }\left(N_{i}\right)\end{array}$ & $\begin{array}{c}\text { Neural Network Structure } \\
\text { No of Hidden layer } \\
\text { Neurons }\left(N_{h}\right)\end{array}$ & $\begin{array}{c}\text { No of Output layer } \\
\text { Neurons }\left(N_{o}\right)\end{array}$ & $\begin{array}{c}\text { Maximum } \% \\
\text { Error }\end{array}$ \\
\hline 1 & 24 & 20 & 12 & -4.61 \\
2 & 24 & 21 & 12 & -4.40 \\
3 & 24 & 22 & 12 & -4.78 \\
\hline
\end{tabular}

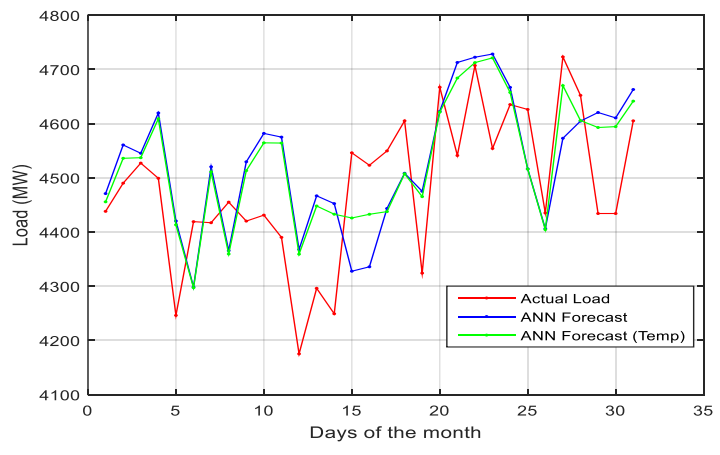

Figure 5. Comparison of actual \& forecasted load curve of ANN model for March 2017

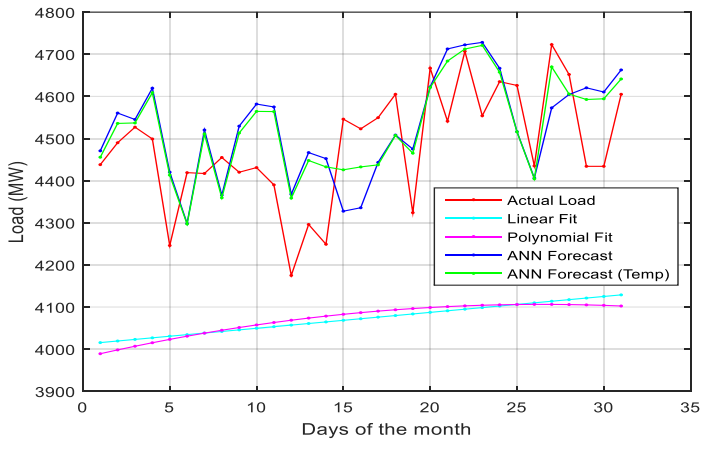

Figure 6. Comparison of conventional \& ANN method for March 2017 


\section{CONCLUSIONS}

The conventional and Artificial Neural Network based approach has been applied for daily peak load forecasting and detailed study has been carried out in terms of training and testing the neural network with historical daily peak load data pertaining to the Bangalore Electricity Supply Company Limited. The ANN model is trained by varying the number of neurons in the hidden layer and it is found that the forecasted values are in good agreement with the actual values for optimal neurons. The ANN model proposed in the present work results in accurate prediction of daily peak load as compared to actual demand. The conventional method gives higher value of error due to nonlinear behavior of most of the loads. Hence the proposed ANN model gives better results in comparison with conventional methods.

\section{ACKNOWLEDGEMENTS}

Authors are grateful to the Pro Vice Chancellor \& Management of School of Engineering \& Technology, JAIN (Deemed-to-be-University)-Bengaluru, the Principal \& Management of Don Bosco Institute of Technology, Bengaluru, the Principal \& Management of M.S. Ramaiah Institute of Technology, Bengaluru, for their constant support and encouragement in carrying out this research work and the BESCOM \& Meteorological center, Bengaluru, for providing the data.

\section{REFERENCES}

[1] Load Generation Balance Report, Government of India, Ministry of Power, Central electricity authority, 2016-17.

[2] IEEE Committee Report, "Load forecasting bibliography". IEEE Trans. on Power Apparatus Systems, vol. 99, no. 1, pp. 53-58, 1980.

[3] Damitha K. Ranaweera, George G. Karady, Richard G. Farmer. "Economic impact analysis of load forecasting," IEEE Transactions on Power Systems, vol. 12, no. 3, pp. 1388-1392, 1977.

[4] Ramesh Kumar V., Pradipkumar Dixit, "One day ahead electrical load forecasting using artificial neural network," $2^{\text {nd }}$ International Conference on Information \& Communication Engineering (IRD India); vol. 2, no. 1, pp. 9-12, Aug 2013.

[5] Tomonobu Senjyu, Hitoshi Takara, Katsumi Uezato, Toshihisa Funabashi, "One-hour-ahead load forecasting using neural network," IEEE Transactions on Power Systems, vol. 17, no. 1, pp. 113-118, Feb 2002.

[6] Vitor Hugo Ferreira, Alexandre P. Alves da Silva, "Toward estimating autonomous neural network-based electric load forecasters," IEEE Transactions on Power Systems, vol. 22, no. 4, pp. 1554-1562, Nov 2007.

[7] Z. A. Bashir, M. E. El-Hawary, "Applying wavelets to short-term load forecasting using pso-based neural networks," IEEE Transactions on Power Systems, 24, no. 1, pp. 20-27, Feb 2009.

[8] Ying Chen, Peter B. Luh, Che Guan, Yige Zhao, Laurent D. Michel, Matthew A. Coolbeth, Peter B. Friedland and Stephen J. Rourke., "Short-term load forecasting: similar day-based wavelet neural networks," IEEE Transactions on Power Systems, vol. 25, no. 1, pp. 322-330, Feb 2010.

[9] Madasu Hanmandlu, Bhavesh Kumar Chauhan, "Load forecasting using hybrid models," IEEE Transactions on Power Systems, vol. 26, no. 1, pp. 20-29, Feb 2011.

[10] Ni Ding, Clementine Benoit, Guillaume Foggia, Yvon Besanger, Frederic Wurtz, "Neural network-based model design for short-term load forecast in distribution systems," IEEE Transactions on Power Systems, vol. 31, no.1, pp. 72-81, Jan 2016

[11] Yizheng Xu, Jovica V. Milanovic, "Day-ahead prediction and shaping of dynamic response of demand at bulk supply points," IEEE Transactions on Power Systems, vol. 31, no. 4, pp. 3100-3108, Jul 2016.

[12] Filip Rodrigues, C. Cardeira, J. M. F. Calado, "The daily and hourly energy consumption and load forecasting using artificial neural network method: a case study using a set of 93 households in Portugal," Elsevier. vol. 62, no. 1, pp. 220-229, 2014.

[13] Anamika Singh, V. Kumar Tripathi, "Load forecasting using multi-layer perceptron neural network," International Journal of Engineering Science and Computing, vol. 6, no. 5, pp. 5463 -5466, May 2016.

[14] Ramesh Kumar V, Pradipkumar Dixit, "Artificial neural network model for hourly peak load forecast," International Journal of Energy Economics and Policy, vol. 8, no. 5, pp. 155-160, Sep 2018.

[15] Pradipkumar Dixit, H. G. Gopal, "ANN based three stage classification of arc gradient of contaminated porcelain insulators," International Conference on Solid Dielectrics, France, vol. 1, no. 1, pp. 427-430, Jul 2004. 


\section{BIOGRAPHIES OF AUTHORS}

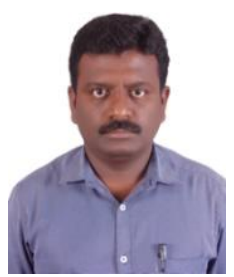

Ramesh Kumar V received the B.E. degree in Electrical \& Electronics Engineering and M.E degree in Power \& Energy Systems from UVCE (Bangalore University), Bengaluru, India, in 2001 and 2004, respectively. He is currently pursuing Ph.D. at JAIN (Deemed-to-be-University), Bengaluru. $\mathrm{He}$ is working as Assistant Professor at Don Bosco Institute of Technology, Bengaluru. His research interest is in the area of Artificial Neural Network and their applications to various Power Systems problems.

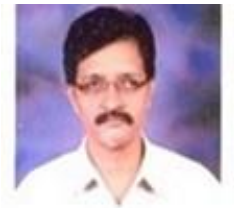

Pradipkumar Dixit received the B.E. degree in Electrical and Electronics Engineering in 1989 from Mysore University, M. Tech in Power and Energy Systems in 1995 from Mangalore University and Ph.D. in High Voltage Engineering in 2009 from Visvesvaraya Technological University, Belagavi. He is currently working as Professor in Electrical \& Electronics Engineering Department of MSRIT, Bengaluru. His areas of interest are high voltage engineering, outdoor insulation, lightning protection, electromagnetic compatibility, and electric power quality. 